\title{
PREPRINT
}

KREJČÍ, Lukáš; SOBOTKA, Jan; NOVÁK, Jiří. Relation Between Test Coverage and Timed Automata Model Structure. In: Tools and Methods of Program Analysis: 5th International Conference, TMPA 2019, Tbilisi, Georgia, November 7-9, 2019, Revised Selected Papers 6. Springer International Publishing, 2021. p. 109-120.

https://www.springerprofessional.de/en/relation-between-test-coverage-and-timed-automata-model-structur/18972558

\section{Relation between Test Coverage and Timed Automata Model Structure} \author{
Lukáš Krejčí1 ${ }^{10000-0002-1733-5654]}, \underset{0782-1285]}{\text { Jan Sobotka }}{ }^{[0000-0002-0275-2025]}$, and Jiří Novák ${ }^{1[0000-0003-}$ \\ ${ }^{1}$ Czech Technical University in Prague - Faculty of Electrical Engineering, Technická 2, Praha \\ 6, 166 27, Czech Republic \\ $\{$ krejclu6, sobotja2, jnovak\}@fel.cvut.cz
}

\begin{abstract}
This paper deals with problematics of structure of Timed Automata models suitable for Model-Based Testing of automotive systems. Previous experiments, primarily focused on the environmental models, have shown that their structure does not significantly affect the coverage speed of testing process. However, similar questions regarding the observer part of the system model remained open. This paper analyzes those remaining questions and focuses on uncovering possible relation between an observer model structure and the quality of generated test sequences according to multiple criteria. Goal of presented experiments is to compare multiple modeling approaches and discover which one is most suitable for automotive systems.
\end{abstract}

Keywords: Timed Automata, Model-Based, Testing, Structure, Coverage, Automotive, Hardware-in-the-Loop, HiL.

\section{Introduction}

In latest decades, requirements for testing of automotive electronics systems during their development are continually rising. Because of increasing complexity of a typical System-under-Test (SUT) and time restrictions induced by limited resources, the testing process itself poses a substantial challenge [1]. As manual design of test cases and test specifications traditionally used in industry practice might lead to various 
subjective errors, the employment of Model-Based Testing (MBT) methods into this process is an asset.

The MBT is a technique of utilization of system and environmental models [2] in order to automatically generate test cases and test suites. This process can be driven by various criteria, characteristically related to the coverage of the SUT state space [3] or to the SUT safety. In order to apply the principles of MBT on the area of integration testing of automotive electronic systems, testing tool Taster [4] was introduced. The modelling language used by Taster is based on Timed Automata network (defined by UPPAAL team [5]) virtually divided into an environment and observer part. The environment part is responsible for providing input stimuli to the SUT, and the observer part monitors the SUT behavior and verifies its correctness.

Nevertheless, introduction of the MBT brings forth new issue - since creating a model of an SUT requires investment of time and effort, it's necessary to create the system model in most appropriate way. Currently, different approaches of modeling of both parts exist. Both the system environment and the SUT itself can be modeled in many ways - from fully permissive models similar to the random generation of test stimuli to entirely restrictive version allowing only specific test cases based on behavioral modeling [6]. Experiments presented in paper [6] have shown that both simple and complex modeling approaches for modeling of SUT environment are comparable in terms of achieved state space coverage. However, the experiments were focused only on coverage criteria and didn't cover the modelling of the observer part. In this paper, four different approaches of environment and observer modelling of real automotive systems are compared and evaluated with respect to multiple criteria

\section{$2 \quad$ Background}

Experiments presented in this paper are based on the same case study used in previous experiments presented in [6], focused on the application of MBT applied on the Hardware-in-the-Loop (HiL) integration testing of the control unit of car trunk doors. The first part of the case study is the SUT model based on the system specification extended by the model of the control unit of car keyless locking system for purpose of experiments presented in this paper. In the second part of the case study, the Taster tool was used for generating and executing test cases on the HiL testing platform, based on the NI VeriStand and NI PXIe and abstracted away by the EXAM testing system. The workflow diagram of the process is shown in Fig. 1. This paper deals with the model part and the question of what the best modelling methodology is for both environment and observer parts

Final paper at https://www.springerprofessional.de/en/relation-between-test-coverage-and-timed-automata-model-structur/18972558 


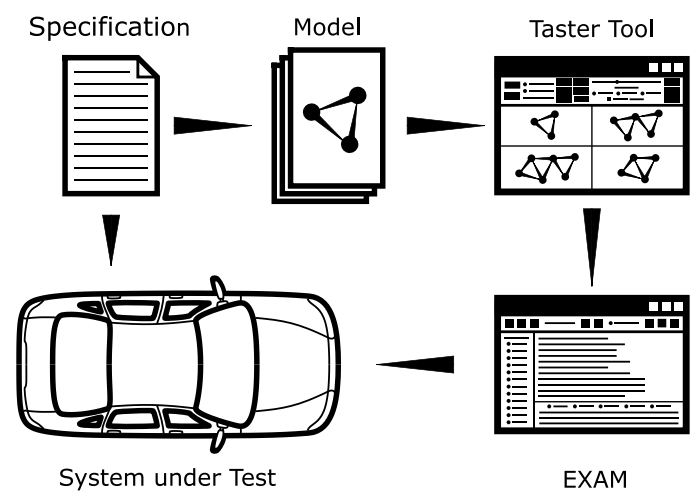

Fig. 1. Diagram of the MBT process using the Taster tool

The case study is focused on two cooperating car subsystems - the trunk doors control unit responsible for operating the automatic trunk doors and keyless locking system control unit responsible for correct function of car's locks. Typical operations performed on the locking system are unlocking and locking the car using the remote control or door handle interaction with remote control in proximity. In the case of the trunk control system, the typical performed operations are opening and closing of automatic trunk and interrupting those operations using one of four trunk control buttons (remote control, dashboard, internal and handle). Inputs of the SUT therefore consist of remote controller position, the door handles, three remote control buttons, one dashboard button and two trunk doors buttons (handle and internal).

During the evaluation of this case study, several questions regarding the modeling techniques has been raised. Questions regarding the environment part of the system models were covered by previous experiments presented in [6]. However, during the experiments, new questions concerning observer part of the model were raised.

Original case study and previous experiments were using a SUT containing only model of the trunk doors control unit. The observer part of the model was created according to the specification of correct behavior of the trunk system and it was fixed for all experiments. While this was sufficient for the purposes of original case study and experiments, it was necessary to find suitable modeling principles for observer part too, once the case study was to be extended with model of the keyless locking system. Similarly to previous situation with environment modeling, multiple approaches for the observer modelling existed and it was not clear, which modeling approach is the best. Consequently, a new set of experiments had to be run.

The experiments presented in this paper are based on the same models as previously. In addition to the two original sets of environment models, two different sets of observer models were created. The first set consists of a single compound observer model and represents the restrictive modelling approach. In the second set, both tested subsystems (the trunk doors control unit and the keyless locking system) have separate synchronized observer models. Both environment models were evaluated with respect to two sets of environment models (restrictive and permissive) used in previous experiments on the HiL testing platform. Test cases were generated by random strategy (selection

Final paper at https://www.springerprofessional.de/en/relation-between-test-coverage-and-timed-automata-model-structur/18972558 
of next edge randomly from a pool of enabled transitions) and systematic strategy (targeting least taken nodes and edges) in online way. The online approach generates test steps directly during runtime as opposed to the offline approach, where test cases are generated in advance.

\section{$3 \quad$ Related Work}

Analysis of MBT approaches, as well measurement of testing efficiency in general, is not straightforward task with standardized methodology. One of possibilities is experimental comparison using some selected criterion or set of criteria. In the field of the MBT, the coverage criteria of a system model are essential parameters [7]. Intuitively, they describe how comprehensively the SUT was tested. Naturally, one of the goals of the MBT [2] is to achieve the best possible model coverage in the minimal time, steps, or similar quantitative measure. Regrettably, there is an infinite number of existing coverage criteria $[8,9]$. This work is based on subset called Structural Model Coverage Criteria [7]. Specifically, coverage of all nodes, all edge, and all pairs of edges (i.e. paths of length of two) are being used in the experiment. Authors of [10] demonstrate, how the structural coverage criteria can be used for test generation using a model checker. The paper [11] reveals that utilization of the structural coverage criteria for supplementary guidance of testing strategies can have a positive impact on the fault detection capability in black-box testing. Additionally, if an SUT model is used in the context of Model-Driven Development, i.e. when the model serves for generation of both SUT source codes and test cases, the paper [12] shows a correlation between coverage of the model structure and coverage of generated code achieved by the generated test cases. Consequently, that shows importance of chosen criteria.

This paper works with the progress of coverage over time, just like previous experiments presented in [6]. This progress is influenced by exploration algorithm and by a model structure. In case of Taster, the model is explored by graph search techniques [13]. Model is divided into environment and observer part, as it was mentioned in the Background section. Observer part is concerned immutable, since it describes system correct behavior. Similarly to works $[14,15]$, the experimental approach was chosen to compare the impact of environment model structure to observer coverage progress. None of the related papers contain results directly comparable with the results presented in this paper.

\section{$4 \quad$ Modeling Language}

As mentioned in Introduction, the developed testing tool is designated for testing of automotive electronics system. Those systems are real-time and reactive, so it was necessary to use modeling language fitting systems with those properties. Hence, the used modeling language is based on the theory of timed automata, developed by UPPAAL team [5], allowing to describe the modeled system as a network of Timed Safety Automata (TSA) bound by a set of variables.

Final paper at https://www.springerprofessional.de/en/relation-between-test-coverage-and-timed-automata-model-structur/18972558 
The original theory of Timed Automata (TA), described in [16], was extended by UPPAAL team into the TSA (described [5]) by introduction of local invariant conditions that ensure progress of each automaton in the system. A single TSA can be formally defined followingly (described in [17]):

- A timed safety automaton $A$ is a tuple $A=\left(N, l_{0}, E, I\right)$, where:

$-N$ is a finite set of locations (i.e. nodes),

$-l_{0} \in N$ is initial location,

$-E \in N \times B(C) \times \Sigma \times 2^{C} \times N$ is the set of edges and

$-I: N \rightarrow B(C)$ assigns invariants to locations.

- We shall write $l \rightarrow g, a, r, l^{\prime}$, when $\left(l, g, a, r, l^{\prime}\right) \in E$.

- A local invariant is a constraint $x<n, x \leq n$, where $n \in \mathbb{N}$.

Informally, TSA is an oriented graph containing states (one of them is initial) and transitions between them. Transitions are labeled by guard condition enabling its execution and priorities affecting the probability of their execution.

A single TSA in an SUT model typically represents a separate model of a specific SUT subsystem and is referred to as a template. An SUT model can contain one or more instances of each template that represent one exact instance of tested subsystem. Because the MBT principles require a model of the SUT environment, multiple TSA are typically used for this purpose, providing input stimuli for the SUT. Interoperation of individual automata within model can be synchronized using system of variables and synchronization channels introduced by the UPPAAL team.

\section{Models}

In order to find most suitable modeling approach, two different model variants were created for both SUT and its environment. Those model variants represent two main modeling paradigms. First approach, referred to as simple, prefers division of the model into multiple simple interoperating automata. Second approach, referred to as complex, prefers utilization of only one, complex automaton. Both of those approaches were applied to modeling of the SUT (car trunk doors and locking systems) and its environment (driver).

\subsection{Observer}

The observer part of the model describes the correct behavior of the SUT according to the specification and its purpose is to ensure correctness of a tested system. Typically, no input stimuli for the SUT are provided by the observer part. In order to verify correctness of the SUT, observer part contains so called invariant conditions checks. In the used modeling language, those invariant conditions are encoded in nodes and are always verified when an automaton enters given state.

Because observer part can cover multiple subsystems of the SUT, the difference between simple and complex approaches primarily lies in separation of individual subsystem. Both observer model variants are described in following subsections.

Final paper at https://www.springerprofessional.de/en/relation-between-test-coverage-and-timed-automata-model-structur/18972558 
Simple approach. In the simple observer approach, referred to as $O b_{S}$, each subsystem is modeled by separate automaton. In the case study used in the experiments, this means that there are two observer models - one for the locking system and one for the trunk control system. The locking system model is shown on Fig. 2.

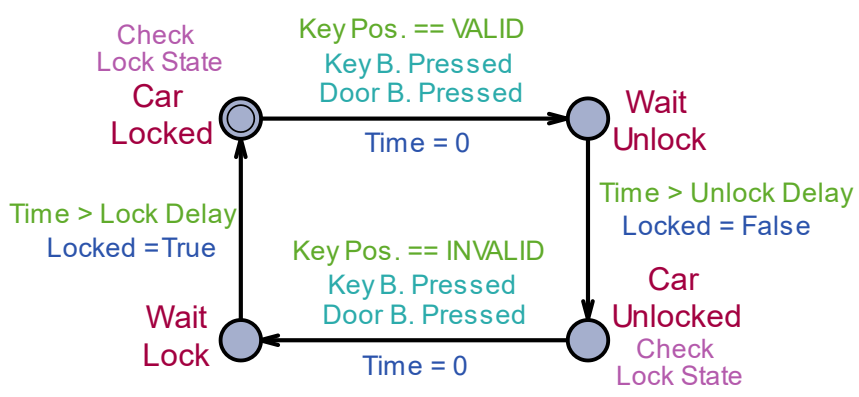

Fig. 2. The locking system observer model.

The trunk control system model is similar to the common observer model used in previous experiments presented in [6] and is shown on Fig. 3.

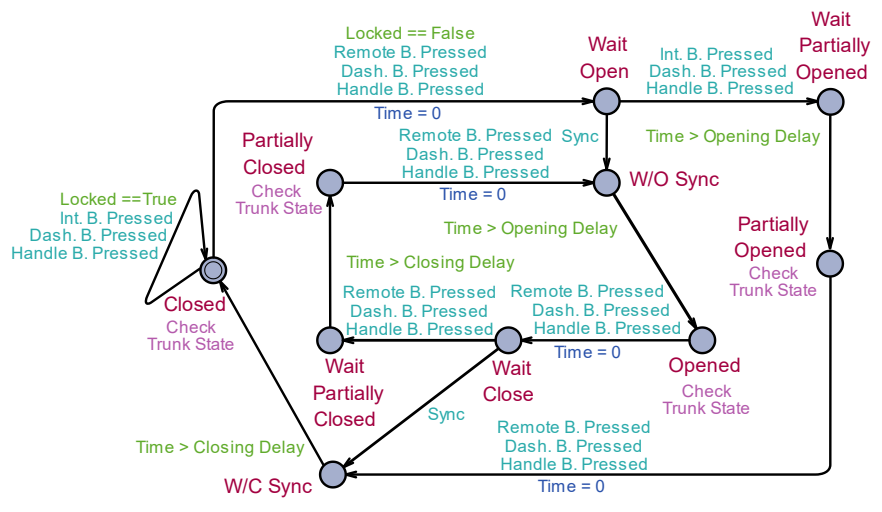

Fig. 3. The trunk system observer model.

Clearly, advantage of this approach lies in its clarity, as it allows to logically divide the SUT model into separate subsystem. Moreover, it allows to capture the parallel nature of a typical SUT. However, it creates additional requirements for synchronization between models of individual subsystems.

Complex approach. In the complex observer approach, referred to as $O b_{C}$, both subsystems are modeled by a single automaton template. Therefore, both models presented in previous section are merged into one describing complex behavior of combined system. The complex observer model is shown of Fig. 4.

Final paper at https://www.springerprofessional.de/en/relation-between-test-coverage-and-timed-automata-model-structur/18972558 


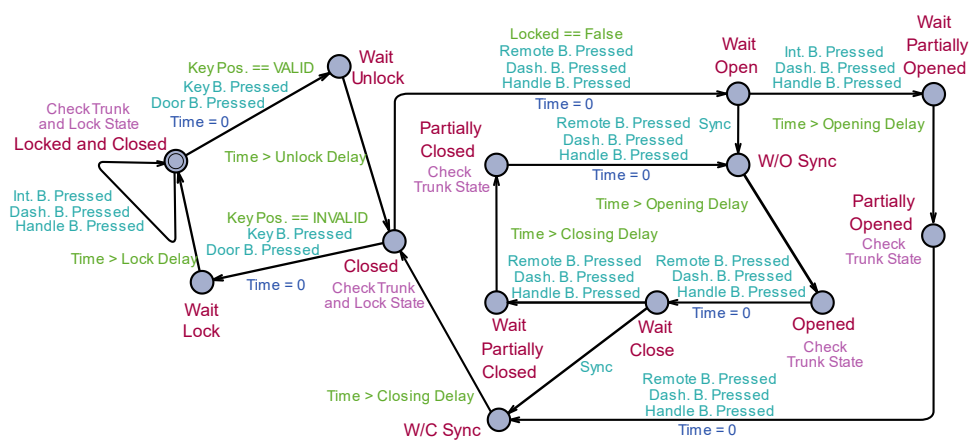

Fig. 4. The complex observer model.

Advantage of complex observer model is the centralization. Unlike in the case of the simple approach, the correct behavior of the SUT is exhaustively described by a single automaton template. Moreover, there is no need for additional synchronization. This approach, however, introduces significant requirements on modeling and bad scalability with increasing number of subsystems. Additionally, this modeling approach fails to capture the parallel nature of the SUT, where multiple subsystems are operated separately.

\subsection{Environment}

In addition to two variants of observer model, two versions of environment models were utilized in the presented experiment. The environmental part of the SUT model represents manipulation with the SUT inputs, i.e. locking and trunk control buttons.

The key difference between both modeling approaches lies within separation of models of individual inputs. Both variants presented in following sections are based on the environment models used in the previous experiments (described in [6]).

Simple approach. The simple environment model, referred to as $E n v_{S}$, introduces a separate automaton instance for each input (i.e. control buttons and remote controller position). Therefore, the full environment model, composed of multiple timed automata instances, represents the set of control inputs. Example of one of the automaton instances is shown in Fig. 5.

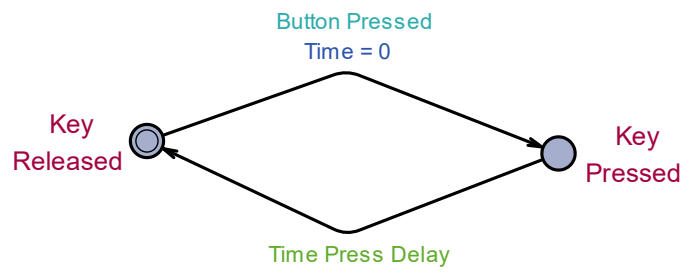

Final paper at https://www.springerprofessional.de/en/relation-between-test-coverage-and-timed-automata-model-structur/18972558 
Fig. 5. Example of a button model in the simple environment model.

Depending on chosen test run strategy (see section Experiment for overview of used strategies), the simple environment model behaves as a car user, who is pressing buttons randomly, systematically or with specific pattern, but always with defined timing.

Undoubtedly, the major advantage of the simple model is its simplicity. Modeling the SUT environment in such way is not overly time consuming and might reduce time required for creating an exhaustive SUT model. Disadvantage of this approach might be creation of high number of unrealistic test cases.

Complex approach. Just like in the case of the complex observer model, the complex environment model, referred to as $E n v_{C}$, consists only from a single automaton instance representing a sensible car user. That means a user, who uses inputs (i.e. pushes buttons and changes position of remote controller) correctly within the given context.

As this automaton represents a car user, it has ability of manipulate with the SUT inputs encoded in the structure of the automaton itself. That additionally allows to add timing information the SUT operations and make the test runs more realistic. The complex environment model is shown in Fig. 6.

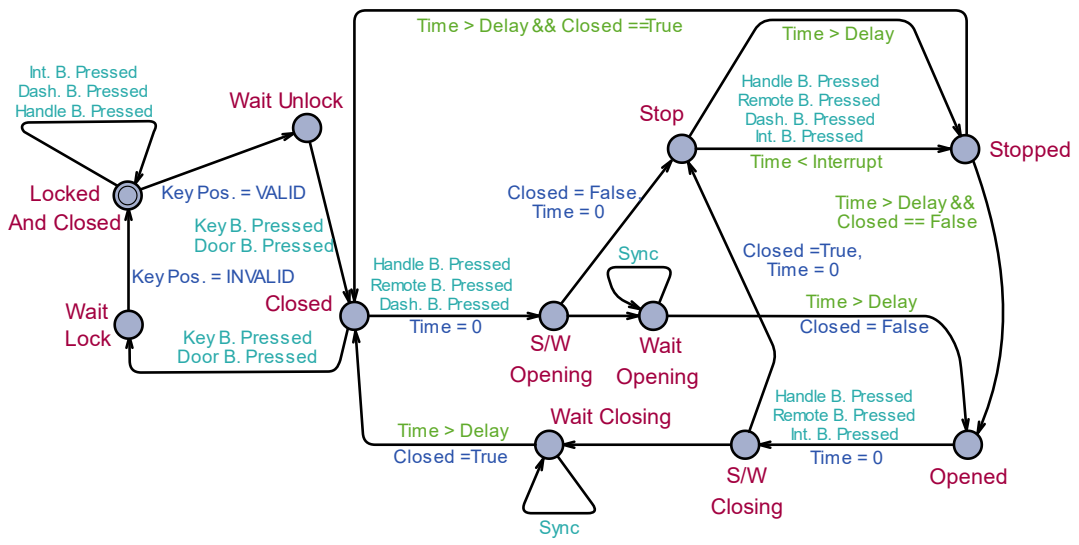

Fig. 6. The complex environment model.

Another advantage of the complex model is ability to utilize Model-Based Statistical Testing (MBST). The MBST is a form of MBT, which additionally uses statistical environment models [18]. Utilization of MBST could potentially provide even more realistic test cases.

\section{Experiment}

The aim of the presented experiments was to compare all combinations of modeling approaches of both observer and environment parts of the SUT model and find the most suitable combination. Because of the varying model structure of individual model

Final paper at https://www.springerprofessional.de/en/relation-between-test-coverage-and-timed-automata-model-structur/18972558 
variants, the structural model coverage was chosen as the primary comparison criteria. Therefore, every modeling variant (i.e. element from the space defined as $\left.\left\{O b_{S}, O b_{C}\right\} \times\left\{E n v_{S}, E n v_{C}\right\}\right)$ was evaluated on the progress of coverage of nodes $\left(C_{N}\right)$, edges $\left(C_{E}\right)$ and pairs of edges $\left(C_{E P}\right)$ over discrete time (i.e. number of executed test steps).

The experimental test runs were executed using the Taster tool. During their execution, the structural coverage data was progressively collected (i.e. $C_{N}, C_{E}$ and $C_{E P}$ for each discrete time point). The tests runs were driven by following strategies:

- Random strategy that choses executed transition randomly,

- Systematic strategy that always choses transition to least visited node, and

- Heuristic strategy that choses the least taken transitions with highest priority.

Results for each modeling variant and each testing strategy were obtained from individual test runs, which duration was limited to one hour. This duration is sufficient, as the length of simulation step in the Taster tool was set to $250 \mathrm{~ms}$, which provides up to 14400 test steps within a single test run.

\section{$7 \quad$ Results}

First evaluated criterion was the node coverage. While the node coverage is just an elementary criterion, it's still essential since the invariant checks are encoded in states of individual automata. Graphs depicting the progress of node coverage for all model variants and all three strategies is shown in Fig. 7.

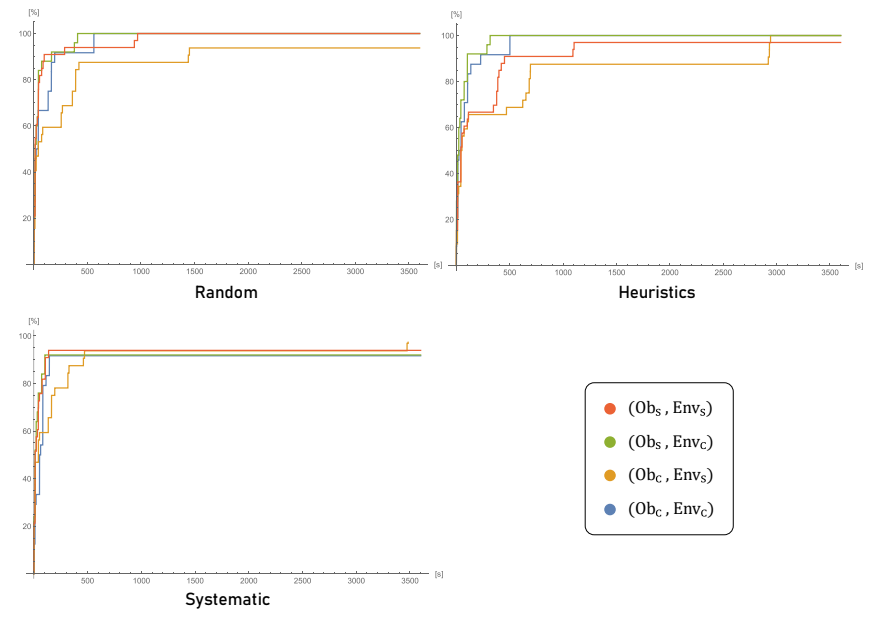

Fig. 7. The node coverage progress.

The evaluated criterion was the edge coverage. Because all actions physically executed with the SUT are bound to the edges in the system model, this criterion is vital. Graph

Final paper at https://www.springerprofessional.de/en/relation-between-test-coverage-and-timed-automata-model-structur/18972558 
depicting the progress of edge coverage for all model variants and all three strategies is shown in Fig. 8.

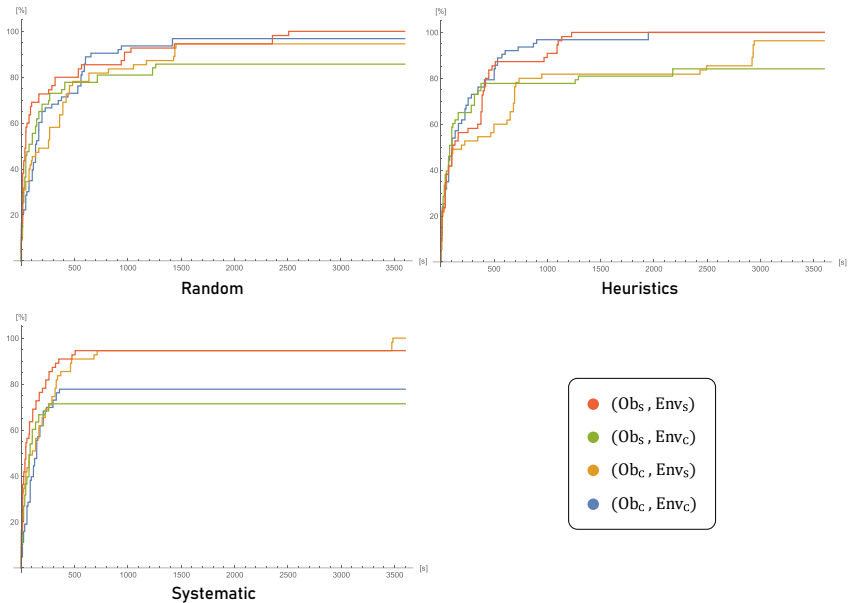

Fig. 8. The edge coverage progress.

Last criterion analyzed in the experiment was the coverage of edge pairs, i.e. coverage of paths of length of two. Since occurrence of some types of faults in the SUT is conditioned by execution of operations in exact order, this criterion can be useful of uncovering of such faults. Graphs depicting progress of the coverage of pairs of edges for all model variants and all three strategies is shown in Fig. 9.

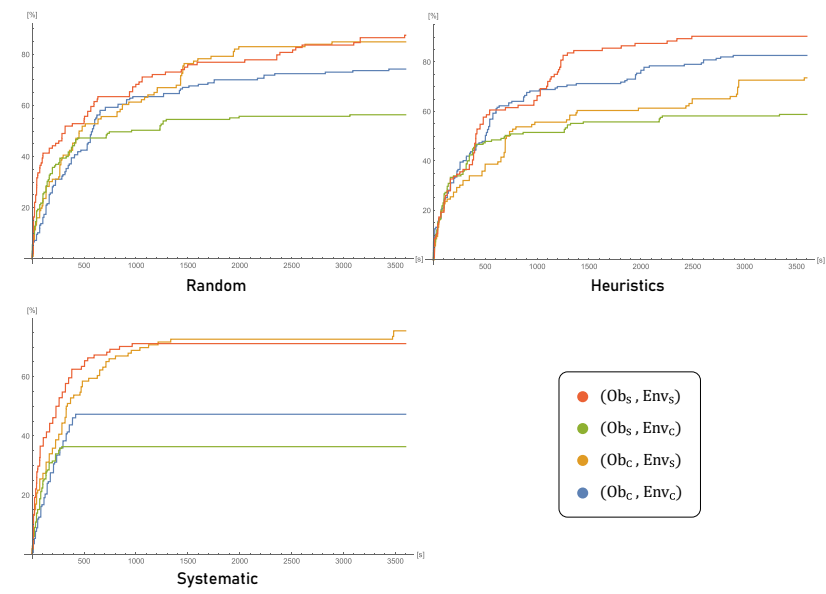

Fig. 9. The edge pairs coverage progress.

All criteria used in above graphs were always obtained from a single test run with given strategy and model variant.

Final paper at https://www.springerprofessional.de/en/relation-between-test-coverage-and-timed-automata-model-structur/18972558 


\section{Conclusions and Future Work}

In this paper, experiment comparing different modeling approaches was presented. The modelling approaches, created as combinations of simple and complex models of both observer $\left(O b_{S}, O b_{C}\right)$ and environment $\left(E n v_{S}, E n v_{C}\right)$ parts of the SUT, are described in details in section Models. The experiment and used SUT models were based on the real automotive system (trunk doors system and locking system). All SUT model variants were compared according to the progress of the structural coverage criteria (node, edge and edge pair coverage) using three different test generation strategies (random, heuristic and systematic).

The acquired data, presented in previous section, show that the combination of $O b_{S}$ and $E n v_{S}$ provides most stable results among all criteria and used strategies. The noticeably worse performance of variants with $E n v_{C}$ in the edge-based criteria is, however, expected, as it is caused by the restrictive nature of the model - realistically modelled driver cannot perform as many operations (and their combination) with the SUT as the permissive model $\left(E n v_{S}\right)$. Additionally, the results suggest that fully complex model variant (using $O b_{C}$ and $E n v_{C}$ ) provides good results as well, with exception of edge-based criteria and the systematic strategy. That is expected, because the $O b_{C}$ was made with real usage of the SUT in mind. Worse results of this variant for edge-based criteria are again caused by restrictive nature of the $E n v_{C}$. The collected data also suggest that the coverage of the observer model depends on the environment model structure, which refutes the hypothesis suggested in [6].

The results show that the most permissive variant (i.e. combination of $E n v_{S}$ and $O b_{S}$ ) provides consistently good results among all used testing strategies and coverage criteria. This implies optimistic conclusion - it is more beneficial to create simpler, divided models, which are significantly easier to create and maintain. The results also suggest that if more realistic test cases are required, the fully restrictive model set (i.e. combination of $E n v_{C}$ and $O b_{C}$ ) should be utilized instead. However, the maintenance of two sets of models is problematic and, as explained before, the worse performance of combination of $E n v_{C}$ and $O b_{S}$ in edge-based criteria is expected. Consequently, more suitable variant is to maintain one simple observer model accompanied by one simple environment model (for test cases with higher coverage) and one driver model (for realistic test cases).

In first part of future research, presented results will be utilized to further expand used case study with additional car subsystems and inputs, such as window control subsystem, propulsion systems status, or intrusion detection. This continually growing case study can be created thanks to the cooperation with our industrial partner. Later, more accurate, behavioral model of car user will be obtained for utilization in the final phase of testing process.

Second part of future research will be primarily focused on the Taster tool and its further development. Presented experiments only utilized three basic strategies. Therefore, it would be desirable to add support of other testing strategies. For example, there is an ongoing research of testing strategy using machine perception and learning. All new testing strategies will be experimentally evaluated on the extended case study.

Final paper at https://www.springerprofessional.de/en/relation-between-test-coverage-and-timed-automata-model-structur/18972558 


\section{Acknowledgement}

This work was supported by the Grant Agency of the Czech Technical University in Prague, projects Optimization methods for Model-Based Testing of automotive systems (grant No. SGS18/144/OHK3/2T/13) and Utilization of machine learning and biologically inspired algorithms in Model-Based Testing of automotive systems (SGS19/071/OHK3/1T/13). This support is gratefully acknowledged.

\section{References}

1. Abelein, U., Lochner, H., Hahn, D., Straube, S.: Complexity, quality and robustness - the challenges of tomorrow's automotive electronics. In: 2012 Design, Automation Test in Europe Conference Exhibition (DATE), pp. 870-871 (2012).

2. Peleska. J.: Industrial-Strength Model-Based Testing - State of the Art and Current Challenges. In: Petrenko, Alexander K. and Schlingloff, Holger (eds.): Proceedings Eighth Workshop on Model-Based Testing, Rome, Italy, 17th March 2013, Electronic Proceedings in Theoretical Computer Science 111, pp. 3-28 (2013).

3. Groote, J. F., Kouters, T. W. D. M., Osaiweran, A.: Specification guidelines to avoid the state space explosion problem. http://dx.doi.org/10.1002/stvr.1536 (2015).

4. Sobotka, J., Novák, J.: Testing Automotive Reactive Systems using Timed Automata. In: Proceedings of the 2017 IEEE 9th International Conference on Inteligent Data Acquisition and Advanced Computing Systems: Technology and Application (IDAACS), pp. 510-513, Ternopil: Ternopil National Economic University (2017).

5. UPPAAL Team, and others: UPPAAL 4.0: Small tutorial, November 2009, http://www.it.uu.se/research/group/darts/uppaal/small_tutorial.pdf, last accessed June 2018.

6. Sobotka, J., Krejčí, L.: Testing of Automotive Systems - Complex vs. Simple Environment Models. In: VALID 2017 The Ninth International Conference on Advances in System Testing and Validation Lifecycle. IARIA (2017).

7. Utting, eds. San Francisco: Chapter 4 - Selecting your tests. In: Practical Model-Based Testing. Pp. $107-137$ (2007).

8. Utting, M., Pretschner, A., Legeard, B.: A Taxonomy of Modelbased Testing Approaches. Softw. Test. Verif. Reliab, 22 (5), 297-312 (2012).

9. Weißleder, S.: Simulated Satisfaction of Coverage Criteria on UML State Machines. In: 2010 Third International Conference on Software Testing, Verification and Validation. IEEE (2010).

10. Rayadurgam, S., Heimdahl, M.P.E.: Coverage based test-case generation using model checkers. In: Eighth Annual IEEE International Conference and Workshop On the Engineering of Computer-Based Systems-ECBS 2001. IEEE (2001).

11. Gay, G., Staats, M., Whalen, M., Heimdahl, M.P.E.: The Risks of Coverage-Directed Test Case Generation. IEEE Transactions on Software Engineering, vol. 41, issue 8, pp. 803-819 (2015).

12. Barasel, A., Conrad, M., Sadeghipour, S., Wegener, J.: The Interplay between Model Coverage and Code Coverage (2003).

13. Aichernig, B. K., Brandl, H., Jöbstl, E., Krenn, W., Schlick, R., Tiran, S.: Killing strategies for model-based mutation testing. Softw. Test. Verif. Reliab, 25: 716-748 (2015).

Final paper at https://www.springerprofessional.de/en/relation-between-test-coverage-and-timed-automata-model-structur/18972558 
14. Gay, G., Rajan, A., Staats, M., Whalen, M., Heimdahl, M. P. E.: The effect of program and model structure on the effectiveness of mc/dc test adequacy coverage. ACM Trans. Softw. Eng. Methodol. vol. 25, pp. 25:1-25:34 (2016).

15. Belli, F., Beyazit, M.: Event-based mutation testing vs. state-based mutation testing - an experimental comparison. In: 2011 IEEE 35th Annual Computer Software and Applications Conference, pp. 650-655 (2011).

16. Alur, R., Dill, D.: Automata for modeling real-time systems. In: Proceedings of the Seventeenth International Colloquium on Automata Languages and Programming, 443(443), 322335 (1990).

17. J., Bengtsson, J., Yi, W.: Timed automata: Semantics, algorithms and tools. Lecture Notes in Computer Science, 3098(316), 87-124 (2004).

18. Böhr, F., Model Based Statistical Testing of Embedded Systems. In: Proceedings of 2011 IEEE Fourth Int. Conf. Softw. Testing, Verif. Valid. Work., pp. 18-25 (2011).

Final paper at https://www.springerprofessional.de/en/relation-between-test-coverage-and-timed-automata-model-structur/18972558 